\title{
Understanding the basic concepts and tools in experimental quantum optics
}

Jorge Arturo Rojas-Santana, Dorilian Lopez-Mago

Jorge Arturo Rojas-Santana, Dorilian Lopez-Mago, "Understanding the basic concepts and tools in experimental quantum optics," Proc. SPIE 10741, Optics Education and Outreach V, 107410T (14 September 2018); doi: $10.1117 / 12.2321658$

EDIE Event: SPIE Optical Engineering + Applications, 2018, San Diego, California, United States 


\title{
Understanding the basic concepts and tools in experimental quantum optics
}

\author{
Jorge Arturo Rojas-Santana and Dorilian Lopez-Mago \\ Tecnologico de Monterrey, Ave. Eugenio Garza Sada 2501, Monterrey, N.L., Mexico, 64849
}

\begin{abstract}
We implement laboratory activities for the construction of key concepts in quantum optics based on experimentation and discussion. These activities were realized in the Quantum Optics Laboratory at Tecnologico de Monterrey with a multidisciplinary group of undergraduate students. We focus on experiments that involve understanding the concept of a photon, such as single photon interference, correlated photons, and entanglement. As an indicator of acquired learning, we design a questionnaire with conceptual questions. This initiative seeks to stimulate the development of multidisciplinary projects in quantum optics taking as a starting point the understanding of basic concepts.
\end{abstract}

Keywords: quantum optics, undergraduate students, experimental laboratory activities, twin-photon source.

\section{INTRODUCTION}

New scientific discoveries transform our vision about nature and shape technological developments. Exciting topics such as quantum computing, quantum information and quantum teleportation, have as scientific support quantum theory. ${ }^{1}$ It is a challenge to transmit to students and interested public the scientific concepts that give support to these possibilities. A strategy to confront this challenge is to structure a thematic that facilitates their understanding.

Many educative activities for the popularization of optics and photonics have been developed since 2015 in the International Year of Light. These activities are dedicated to the importance of sciences and technologies based on light in our daily life. ${ }^{2}$ The level of the activities ranges from very basic demonstrations to more complicated experiments. In particular, we focus our attention in quantum technologies where quantum optics is important.

The manipulation of individual atoms and photons is the basis of quantum technologies such as quantum atomic clocks, quantum sensors, quantum computers, among others. The development of quantum technologies is of great interest, for example the European commission will launch 1 billion quantum technologies flagship. ${ }^{3}$ Mexico considers the development of quantum optics through the National Laboratory of Quantum Matter: Ultracold Matter and Quantum Information and in The Mexican Photonics Initiative. ${ }^{4}$

We consider that the incorporation of quantum technology principles in the educative system is a challenge to motivate their study as a multidisciplinary science. In this work, we propose an educative strategy to structure a thematic about quantum optics based on experimentation and discussion. We analyze the student reactions working in a twin-photon source at the quantum optics laboratory at Tecnologico de Monterrey. Additionally, we report the main learnings of the students based on their laboratory reports.

In the second section we present the facilities of our research group to work with experiments for undergraduate students. In section three, we formulate an activity about the characterization of a twin-photon source to study the concepts of coincidence, correlated photons, photon polarization and histogram of coincidences. We present the results and discussion in section four and finally the conclusions.

Further author information:

Dorilian Lopez-Mago: E-mail: dlopezmago@itesm.mx, Telephone: +52 (81) 8358-2000

Jorge Arturo Rojas-Santana: E-mail: A00825456@itesm.mx, Telephone: +52 (81) 8358-2000

Optics Education and Outreach V, edited by G. Groot Gregory, Proc. of SPIE Vol. 10741, 107410T (C) 2018 SPIE · CCC code: $0277-786 X / 18 / \$ 18 \cdot$ doi: 10.1117/12.2321658 


\section{PHYSICAL OPTICS GROUP LABORATORY}

The Physical Optics Group provides laboratory practices for students of physical engineering, development of applied engineering projects, basic and applied research, workshops of optical tools and dissemination of science, Fig. 1.

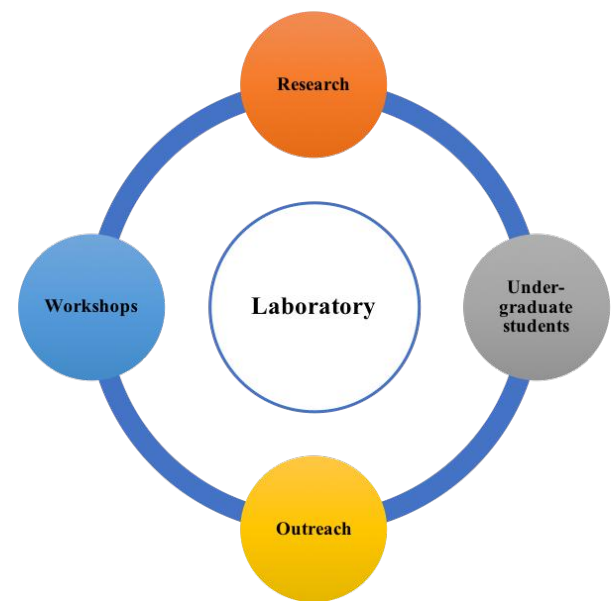

Figure 1. Pictorial of the activities and services provided by the quantum optics laboratory.

The integration of laboratory into multiple activities gives the opportunity to interact with students of multiple disciplines and proposed projects in the area of optics and photonics. The principal research projects are:

- Optical Forces in optical beams with space-variant polarization

- Geometric phase polarimetry

- Quantum Optical Coherence Tomography (QOCT)

- Terahertz optical elements fabricated by 3D-printing technology

Recently, our laboratory established a collaboration with the National Laboratory of Quantum Matter: Ultracold Matter and Quantum Information. This laboratory seeks to be a center of excellence in the country that links selected tasks of basic and applied research, teaching and outreach between national and international groups. Following these objectives, we analyze an optical setup that implements a twin-photon source, which is part of the QOCT experiment. This provides undergraduate students the possibility to work with current scientific projects.

\section{QUANTUM OPTICS EXPERIMENTS FOR UNDERGRADUATE STUDENTS}

There is an academic initiative to promote quantum physics in the undergraduate level, principally computational activities for the visualization and simulation of quantum phenomena. ${ }^{5-7}$ Experimental activities in quantum optics is a challenge, principally for the required expensive instruments. However, it is important to make an effort and bring to the students the opportunity to work in experimental quantum optics. We find interesting the experiments for undergraduate students proposed by Galvez and Scholz on quantum interferometry with individual photons where a twin-photon source is necessary and open the window to advanced quantum optics experiments. $^{8,9}$

A twin-photon source is a non-classical source of light which produce pairs of photons through the spontaneous parametric down conversion (SPDC). In this source a pump beam with frequency $\omega_{p}$ hits a non-linear crystal (NLC), one of the photons can be absorbed and converted into a pair of entangled photons $\omega_{s}$ (signal) and 
$\omega_{i}$ (idler) by the process of spontaneous parametric down conversion (SPDC). Crystals type I produce timefrequency entangled photons and type II produce polarized entangled photons. The phase matching conditions $\omega_{p}=\omega_{s}+\omega_{i}$ determine the trajectory of the photons, i.e. the angle of emission. Fig. 2 shows a schematic of the SPDC experiment.

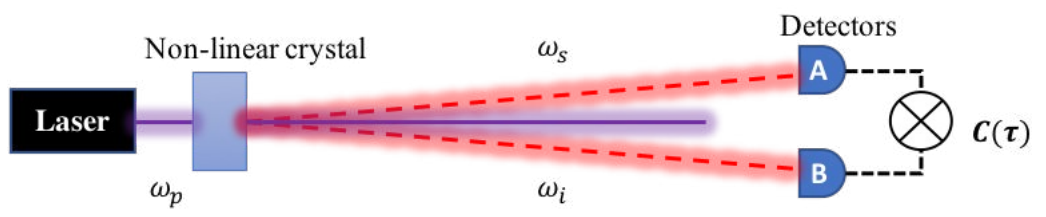

Figure 2. Basic twin-photon source components. A blue laser with central wavelength of $405 \mathrm{~nm}$ pumps a non-linear BBO crystal. The crystal optics axis and pump polarization are arranged to produce SPDC type I photons at 810 nm. The detection system consists of a pair of photodetectors and coincidence electronics.

The detection of this pair of photons requires highly efficient detectors and coincidence counter electronics. The photon coincidences $C(\tau)$ give information about the photon pair production. With this source is possible to experimentally study the concepts of photons, correlated photons, photon polarization, photon coincidences and histogram of coincidences.

\section{ACTIVITY: CHARACTERIZATION OF A TWIN PHOTON SOURCE}

We design the activity about the characterization of a twin-photon source with the objective to know how the students understand the basic concepts and instruments that conform an experimental laboratory of quantum optics. The activity consists in three steps: explanation, experimentation and discussion.

In the explanation, the instructor focuses in the details of Fig. 2 and gives a background about the pump laser, non-linear crystal, SPDC and photons coincidences. Technical details about the manipulation of the equipment and cautions such as the use of security glasses. The experimentation consists in the alignment of the detection system of the twin-photon source, measure the photon coincidences and calculate the joint current rate (JCR) given by $J C R=$ photoncoincidences $/ s P_{p}$, where $P_{p}$ is the laser pumping power.

We apply this activity to multidisciplinary and physics students. For multidisciplinary students we emphasize in the concepts of photon, correlated photons and photon coincidences. The hands-on activity includes the measurement of the coupling efficiency in the optical fibers of the alignment system, as shown in Fig. 3.
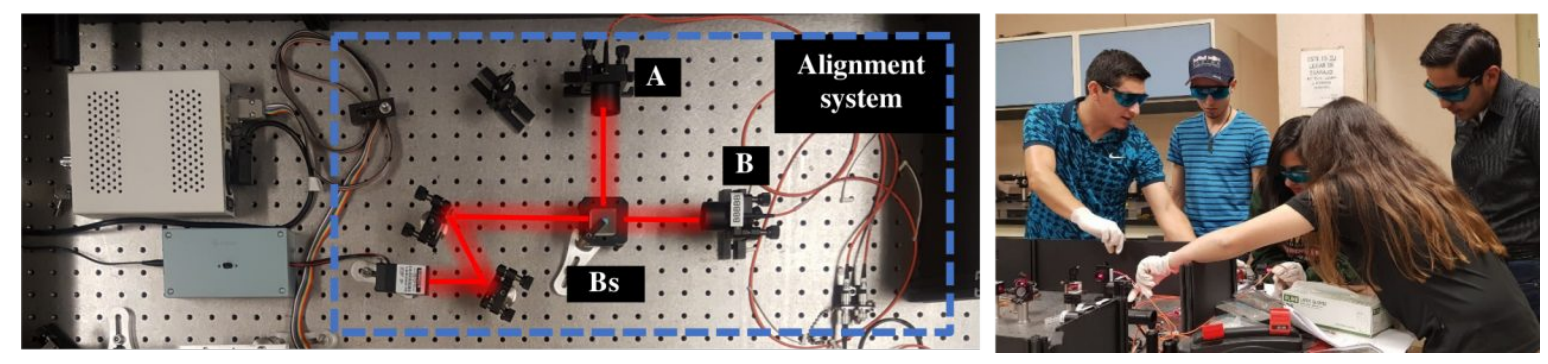

Figure 3. Alignment system. It consists of a $637 \mathrm{~nm}$ laser, beam splitter, fiber optic couplers A and B. Multidisciplinary group of students working in the Quantum Optics Laboratory.

In the case of the group of physics students, we emphasize in the concepts of photon, correlated photons, photon polarization, photon coincidences and histogram of coincidences, Fig. 4.

In this activity students align the detectors mounted in a motorized stage with the purpose to obtain a better signal. The students analyze the photon coincidences versus pump power and determine the JCR. Additionally, they study the effect of an electronic delay, induced adding cable in the detector B (stop). Finally, one polarizer in front of a detector is crossed to observe what happen with the coincidences. Students discuss in the lab 

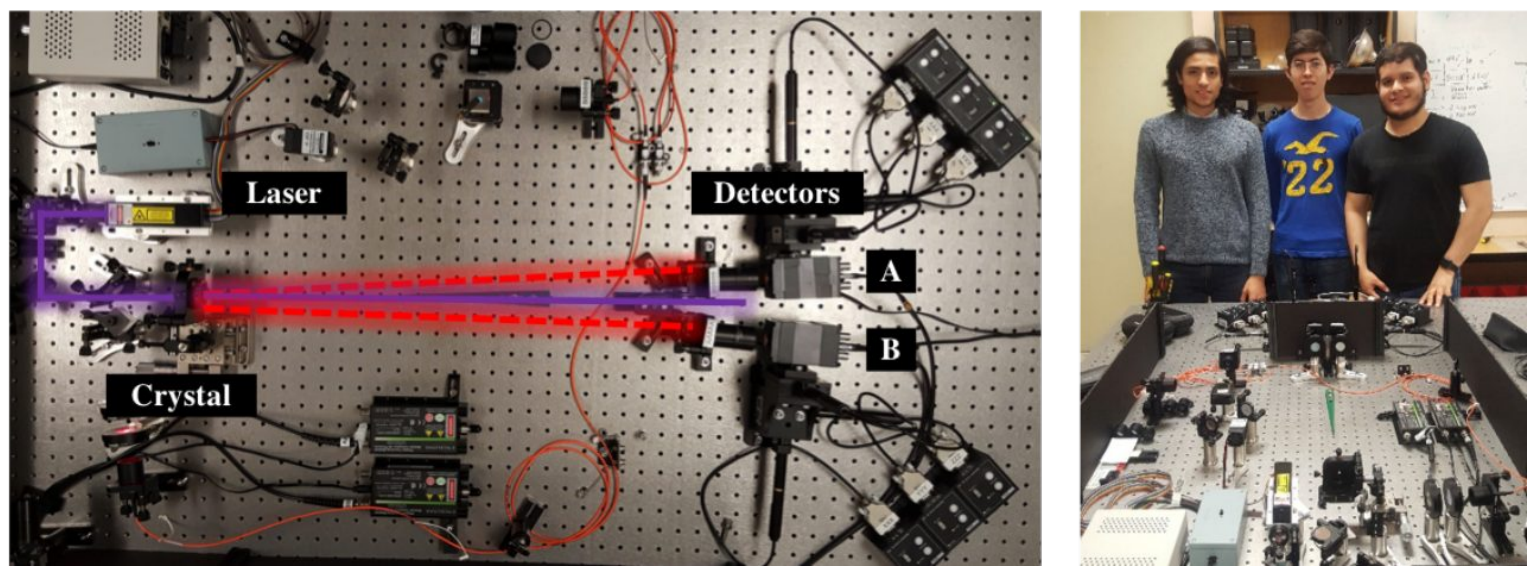

Figure 4. Twin-photon source components. Pumping laser with central wavelength of 405nm, BBO crystal, photodetectors (MPD) in motorized stage A and B. Physics students.

with the instructor the observations and finally present a report. The complete activity can be consulted in https://dorilian.com/laboratory/.

\section{RESULTS}

Students presents high expectation about the experimentation in the laboratory of quantum optics. For the multidisciplinary group of students was a surprise the use of glasses and gloves in the laboratory, one of them study medicine and this gives him security in the activity. After putting on the laser in the alignment system, mentally the students trace the optical path of the beam and when they see how the optical fiber guide the ray they were surprised. Then, the instructor misaligns the fiber and the students take the challenge to optimize the coupling efficiency of the optical fiber, as shown in Fig. 3.

After approximately twenty minutes, the students achieved an efficiency of 90 percent measuring the input power $P_{\text {in }}$ and output power $P_{\text {out }}$ employing a power meter and calculating $P_{\text {out }} / P_{\text {in }}$. The procedure to optimize the coupling efficiency in the optical fiber is similar to the alignment of the detectors $\mathrm{A}$ and $\mathrm{B}$ in Fig. 4 . If we consider a power of $50 \mathrm{~mW}$, the number of photons with wavelength of $810 \mathrm{~nm}$ per second is approximately $2 \times 10^{17}$ and if the idea is to detect individual photons we need high sensitivity of detectors and movements with high precision to do it.

The photon detection is visualized as an electrical pulse in an oscilloscope, as shown Fig. 5. Most students have never used an oscilloscope and the instructor have to show how it works. The instructor programs the channel A to photon detection and the students reproduce the procedure in channel $\mathrm{B}$. The signal in both channels are saved and processed to count the number of coincidences.

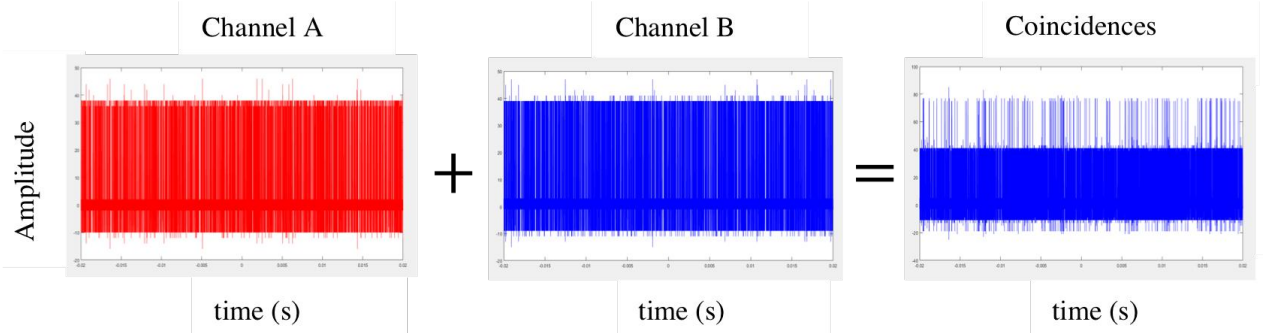

Figure 5. Signal of photon detection in an oscilloscope. Frequency signal of $63 \mathrm{kHz}$ in both channels (A and B). The superposition of the signals result in 184 coincidences of correlated photons. 
Summing the signals in channel A and B the students can visualize the simultaneous pulses which is the result of a pair of coincidences detected. Under this method the concept of coincidence is controversial because changing the time resolution in the oscilloscope, the students observe a difference of some nanoseconds between the "simultaneous coincidences". This helps to understand that we need a faster instrument to detect a pair of coincidences.

The interest an initiative of this multidisciplinary group of students facilitates the work in the lab where the planned activity allows an easy first approach to understand the concepts and tools implemented in quantum optics. This activity allowed us to know the reaction of students about quantum optics experiments and we realized that working in a less complex activity but analogous to one of quantum optics creates expectation in the student and allows him to play with greater confidence in the laboratory. Choosing the activity of coupling optical fiber is very productive to achieve this purpose.

The activity for the group of physics students consists in the analysis of photon coincidences vs pump power. We use a Time-Correlated Single Photon Counting (TCSPC) system (PicoHarp 300) and photon counting detectors (MDP) for the photon detection which is specialized equipment for quantum optics experiments. The detection of photon pairs stars characterizing the dark counts which are intrinsic counts in the detectors using the PicoHarp software. After setting the pump laser at $100 \mathrm{~mW}$ the signal increases and the students must align the detectors mounted in a motorized stage with the purpose to optimize the signal. Students manage this challenge and get expertise in the use of motorized stage, they start with levels of $5 k$ counts per second (cps) and finish with $300 k \mathrm{cps}$, this was a record in our lab with these detectors. For the detection of twin photons, the detectors (A and B) are fixed approximately with same cps. After one hundred seconds of integration time a histogram of coincidences is recorded, it represents the count of correlated photons emitted by the source, as shown in Fig. 6a. The students measure the dependence of coincidences changing the pump power, Fig. $6 \mathrm{~b}$.
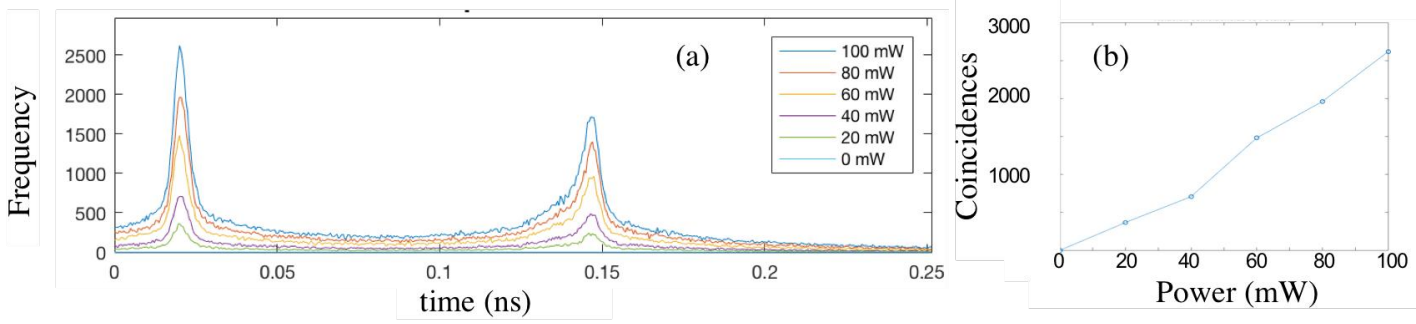

Figure 6. Figure adapted from a student report. a) histogram of coincidences and b) coincidences vs pump power.

The signal presents two peaks of coincidences, some observations of the students are:

- Entre mayor es la potencia, mayor es el nmero de coincidencias (the greater the power, the greater the number of coincidences).

- Entre ms altura tiene el punto, significa que ms coincidencias tuvo en ese determinado tiempo (the higher the point, the more coincidences it had at that time).

- La tasa de pares de fotones varia muy poco y se puede decir que se mantiene constante, esta en realidad es la pendiente (the rate of photon pairs varies very little and can be said to remain constant, this is actually the slope).

Adding $6 \mathrm{~m}$ of cable to detector B, we observe a delay of the peaks, Fig. 7 .

An explanation of the students is: al alargar el cable un pulso tarda ms en llegar y por lo tanto la diferencia de tiempo detectado se alarga (by lengthening the cable a pulse takes longer to arrive and therefore the time difference detected is lengthened).

Finally, some conclusions of the students about the activity are: 


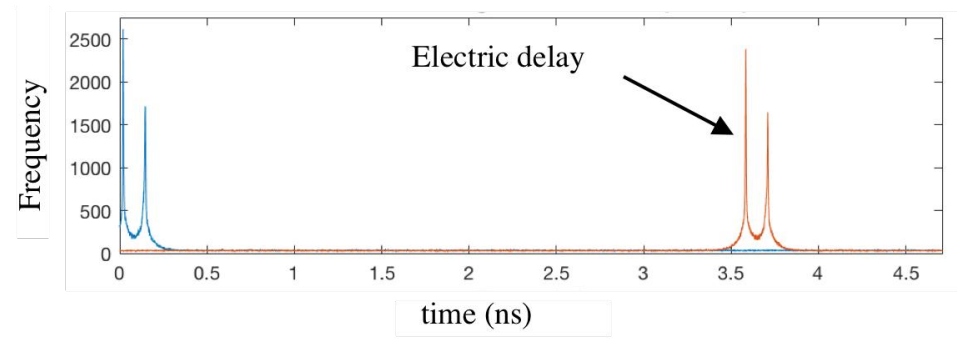

Figure 7. Temporal shift of the peak of coincidences (figure adapted from student report).

- Aprendimos acerca de procedimientos experimentales de optica cuntica (we learned about experimental procedures of quantum optics).

- Aprendimos de la amplia gama de opciones que tenemos para monitorear y realizar cierto tipo de experimentos (we learned the wide range of options that we have to monitor and perform certain tyoes of experiments).

- Reforzo nuestros conocimientos acerca de los diferentes componentes del laboratorio (reinforced our knowledge about the different components of the laboratory).

- A los integrantes del equipo nos tomo un tiempo considerable el acostumbrarse a la obscuridad y moverse con seguridad dentro del laboratorio (the team members took a considerable amount of time to get used to the dark and move safely inside the lab).

- La practica fue interesante debido a que estudiamos fuentes de luz no clasica (the practice was interesting because we studied non-classical light sources).

- Obtuvimos un primer acercamiento a la experimentacion cuantica (we obtained a first approach to quantum experimentation).

- En el experimento el fotn se definir como el pulso que representa una coincidencia de pares de fotones en un determinado diferencial de tiempo (in the experiment, the photon will be defined as the pulse that represents a coincidence of pairs of photons in a given time differential).

- Cuando la diferencia de tiempo es muy prxima a cero se dice que tenemos un par de fotones correlacionados (when the time difference is very close to zero it is said that we have a pair of correlated photons).

- El cristal BBO al ser un medio activo no lineal permite dividir un foton en dos, por el proceso SPDC (the BBO crystal being a non-linear active medium allows one photon to be divided into two, by the SPDC process).

- Fuente de luz no clsica es aquella que permite la generacin discreta de fotones (non-classical light source is one that allows the discrete generation of photons).

- El objetivo de la fuente no clsica usada aqu es emitir un nmero controlado de fotones con una frecuencia especfica (the objective of the non-classical source used here is to emit a controlled number of photons with a specific frequency).

The students complete successfully the experimental activities and their principal learnings were about the use of lasers, mirrors, beam splitters, fiber optics, couplers, power meter, optical enclosure, optical table, detectors APD and MDP, non-linear crystals, oscilloscope, TCSPC system, motorized stage, glasses and gloves. On the other hand, the concepts of photon, time-frequency entangled photons, correlated photons, histogram of coincidences are studied experimentally. 


\section{CONCLUSIONS}

The design of laboratory activities about the characterization of a twin-photon source under the strategy of explanation, experimentation, and discussion is very enriching to understand the function of the experimental devices in a quantum optics laboratory. In the case of students from an area different from physics, it was agreed to perform an experimental work with a lower degree of difficulty. In our case, coupling light to an optical fiber results in a very didactic activity and benefits by being an important part for the alignment of the twin-photon source. On the other hand, for the group of students with major in physical engineering who worked on the characterization of the source of pairs of photons, they had a formal approach to experimental quantum optics.

The proposed dynamic allows the student to obtain an empirical knowledge when discussing situations and doubts in the laboratory. For a case like the group of students of physical engineering, a feedback after the preparation of their report would reinforce the knowledge acquired and clarify doubts. The construction of a twin photon source opens the possibility to do the activities proposed by Galvez ${ }^{8}$ and Scholtz. ${ }^{9}$

\section{ACKNOWLEDGMENTS}

Consejo Nacional de Ciencia y Tecnologia (CONACYT) (Grants 257517, 280181) and Red de Tecnologias Cuanticas.

\section{REFERENCES}

[1] Zeilinger, A., [Dance of the Photons: From Einstein to Quantum Teleportation] (2010).

[2] "2015 International Year of Light and Light-Based Technologies." http://www.light2015.org/Home.html. (Accessed: 11 June 2018).

[3] De Touzalin, A., M. C. H. F. C. I. M. R. and Calarco, T., "Quantum manifesto. A new era of technology. European Comission.," (2016).

[4] Oracio Cuauhtemoc Barbosa Garcia, Luis Alfonso Lastras Martinez, E. R. M. M. J. M. M. B. L. A. O. G. J. S. R. S. and Solis, E. R., "TOWARDS A BRIGHTER MEXICO: OPTICS AND PHOTONICS ROADMAP," tech. rep., Ministery of Economy (2014).

[5] Schroeder, D. V., "Physics Educational Software for Your Browser." 13 March 2017. http://physics. weber . edu/schroeder/software/. (Accessed:23 June 2018).

[6] "PhET Interactive Simulations." 2018. https://phet.colorado.edu/. (Accessed: 28 June 2018).

[7] Johansson, P., "QuTiP - Quantum Toolbox in Python." 2013 and later. http://qutip.org/tutorials . html. (Accessed: 22 June 2018).

[8] Galvez, E. J., Holbrow, C. H., Pysher, M. J., Martin, J. W., Courtemanche, N., Heilig, L., and Spencer, J., "Interference with correlated photons: Five quantum mechanics experiments for undergraduates," American Journal of Physics 73(2), 127-140 (2005).

[9] Scholz, R. and Friege, G., "Undergraduate quantum optics : experimental steps to quantum physics Undergraduate quantum optics : experimental steps to quantum physics," Eur J Phys 39, 055301 (2018). 